This item was submitted to Loughborough's Research Repository by the author.

Items in Figshare are protected by copyright, with all rights reserved, unless otherwise indicated.

\title{
Investigating optimal technique in the presence of motor system noise: application to the double layout somersault dismount on high bar
}

\section{PLEASE CITE THE PUBLISHED VERSION}

http://dx.doi.org/10.1080/02640414.2015.1057211

\section{PUBLISHER}

(C) Taylor \& Francis

\section{VERSION}

AM (Accepted Manuscript)

\section{PUBLISHER STATEMENT}

This work is made available according to the conditions of the Creative Commons Attribution-NonCommercialNoDerivatives 4.0 International (CC BY-NC-ND 4.0) licence. Full details of this licence are available at: https://creativecommons.org/licenses/by-nc-nd/4.0/

\section{LICENCE}

CC BY-NC-ND 4.0

\section{REPOSITORY RECORD}

Hiley, Michael, and Fred Yeadon. 2015. "Investigating Optimal Technique in the Presence of Motor System Noise: Application to the Double Layout Somersault Dismount on High Bar”. Loughborough University. https://hdl.handle.net/2134/18646. 


\title{
Investigating optimal technique in the presence of motor system noise: application to the double layout somersault dismount on high bar
}

\author{
Michael J. Hiley ${ }^{1}$ and Maurice R. Yeadon ${ }^{1}$ \\ ${ }^{1}$ School of Sport, Exercise \& Health Sciences, Loughborough University, Loughborough, UK
}

\begin{abstract}
Minimising joint torque is often used as an optimisation criterion when investigating human movement. Alternatively, an aspect of performance may be chosen to be maximised when investigating sporting movements. The aim of the study was to optimise the technique in the backward giant circle prior to a double layout somersault dismount from the high bar using various criteria to determine which best characterised the technique adopted by a gymnast. Ten recorded gymnast trials were used to determine bar release parameters and the level of noise in the gymnast's movements. A computer simulation model of a gymnast and bar was used to optimise giant circle technique under three criteria: minimising joint torques, maximising the release window and maximising success in the presence of motor system noise. Local and global optimisations of technique were performed using the three criteria starting from the average technique of the 10 recorded trials. All global optimum solutions diverged from the gymnast's technique. The local optimum for maximising success in the presence of noise had a success rate comparable with the global optimum ( $98 \%$ vs. $100 \%$, respectively). It is concluded that the gymnast's technique is characterised by maximising success despite operating with motor system noise.
\end{abstract}

Keywords: simulation, optimisation criterion, movement variability, gymnastics

\section{INTRODUCTION}

Minimising effort (joint torques) or energy has been used to describe the underlying technique of everyday activities such as walking (Ren, Jones \& Howard, 2007; Anderson \& Pandy, 2001). When optimising sporting movements minimising joint torque has also been used with varying degrees of success (Hiley \& Yeadon, 2012; Koschorreck \& Mombaur, 2012; Yamasaki, Gotoh \& Xin, 2010). Wolpert (2007), however, proposed that maximising the likelihood of success at a task, despite the presence of noise within the motor system, was a more plausible explanation of technique in human movement. Therefore, does an optimisation criterion based on maximising the likelihood of success in the presence of noise provide a better characterisation of the technique adopted by athletes than criteria based on minimising joint torque or maximising some other biomechanical descriptor?

In order to maximise success, an understanding of the movement and a clear definition of success are required. It has been demonstrated that in addition to strength, one of the limiting factors to producing angular momentum in backward giant circles (Figure $1 \mathrm{a} \& \mathrm{~b}$ ) prior to a dismount (Figure $1 \mathrm{c}$ ) is the gymnast's ability to time the release from the bar (Hiley \& Yeadon, 2005). The greater the angular momentum produced by the gymnast the smaller the release window became, where the release window was defined as the time interval for which the gymnast had sufficient linear and angular momentum to complete the dismount. A suitably large release window is required otherwise the gymnast will be unable to time the release with sufficient accuracy. 


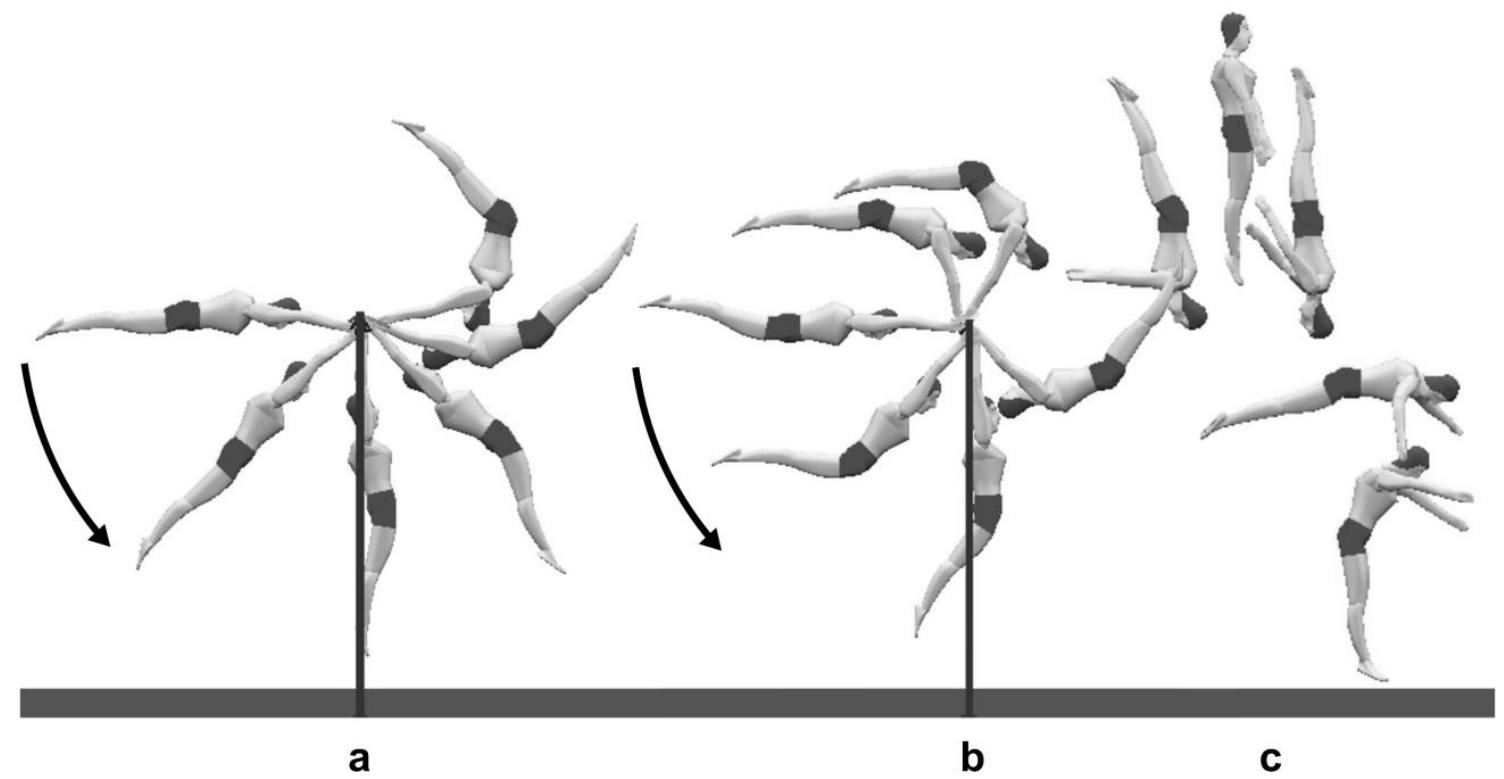

Figure 1. Graphics sequence of (a \& b) one and a half backward giant circles preceding (c) a double layout somersault dismount from high bar.

In addition to producing a suitably large release window and remaining within realistic strength limits, the backward giant circle technique must also be robust to small errors in timing the joint actions. Optimal technique is often sensitive to small errors; therefore introducing small timing errors into optimal giant circle technique can lead to simulations with unrealistically small release windows (Hiley \& Yeadon, 2008). It has been shown that the variability observed in a pointing task (errors in end-point location) was largely due to noise in the execution stage of the movement rather than errors in the localisation (estimation of initial conditions) and planning stages (van Beers, Haggard \& Wolpert, 2004). Given that human movement will always contain some level of variability, since there will always be inherent noise from the motor system (Bartlett, Wheat \& Robins, 2007; Cohen \& Sternad, 2009; Newell \& Corcos, 1993), it would make sense if gymnasts developed robust techniques that produce viable release windows on every attempt. Success in giant circles prior to a dismount would therefore be defined as having a sufficiently large release window, operating within realistic strength limits and being robust in the presence of motor system noise.

More recently it has been demonstrated that the underlying strategy for gymnasts performing the upstart on the uneven bars may be explained by attempting to maximise success at the task despite the presence of noise in the gymnast's movements (Hiley \& Yeadon, 2013). However, it is unclear whether maximising success can explain the technique used in more complex skills such as the giant circles performed prior to a double layout somersault dismount.

The aim of the present study is to determine what optimisation criterion best characterises the technique adopted by a gymnast in the backward giant circles prior to release for a double layout dismount. It is hypothesised that a criterion based on maximising success at the task despite the presence of noise from the motor system will provide a better description of the underlying technique compared to criteria based on minimising joint torque or maximising the release window. 


\section{METHODS}

\section{Data collection}

One male gymnast (age 24 years, mass $70 \mathrm{~kg}$, height $1.73 \mathrm{~m}$ ) who competed internationally gave informed consent to participate in the study which was approved by the University's Ethical Advisory Committee. The gymnast performed 10 successful double layout somersault dismounts from the high bar on to landing mats from 10 attempts which were captured using 15 Vicon MX13 cameras operating at $300 \mathrm{~Hz}$. All trials were observed and approved by an International Brevet judge. Ten trials were chosen to provide a representative spread of the gymnast's performances (James, 2004; Hamill, van Emmerik, Heiderscheit \& Li, 1999). Spherical reflective markers, $25 \mathrm{~mm}$ in diameter, were attached to the lateral side of the wrist, elbow, shoulder, hip, knee and ankle joint centres and toes on the left side of the body. Lateral offset measurements from each marker to the adjacent joint centre were recorded for subsequent location of the joint centres. Additional markers were attached to each side of the gymnast's head (above the ear) and to the centre of the high bar. Prior to data collection a volume centred on the high bar spanning $2 \mathrm{~m} \times 5$ $\mathrm{m} \times 5 \mathrm{~m}$ was wand calibrated using the motion analysis system. The root mean squared reconstruction error for each camera was less than $1 \mathrm{~mm}$. Threedimensional marker coordinates were reconstructed and joint centre locations were determined using the measured offsets. The data in each trial for the last 1.5 giant circles and the aerial phase of the dismount were analysed (Figure 1).

An estimate of the level of noise within the gymnast's joint angle time histories was determined from the measures of kinematic variability during the 10 high bar circles (Hiley, Zuevsky \& Yeadon, 2013). The temporal and spatial noise was defined as the smallest standard deviation at the eight turning points between flexion and extension at the shoulder and hip (i.e. four turning points at the shoulder and four at the hip) during the 1.5 giant circles before release (Figure $2 a$ and Table 1). The lowest measure of variability was chosen to represent execution noise (van Beers et al., 2004). Although kinematic variability does not arise solely from motor system noise (e.g. may contain variation due to feedback control and covariation), in tasks that require precise timing it has been shown that kinematic variability is minimised in the important actions (Broderick \& Newell, 1999; Hiley et al., 2013). If the gymnast has attempted to minimise kinematic variability then the measured values at the extrema (Hiley et al., 2013) will therefore have a large component due to noise (Cohen \& Sternad, 2009).

Table 1. Standard deviations in the temporal and spatial dimensions at the extrema of the shoulder and hip time histories for the 10 double layout trials from Hiley et al. (2013)

\begin{tabular}{ccccccccc}
\hline Standard & Hip & Hip & Hip & Hip & Shld & Shld & Shld & Shld \\
deviation & Max & Min & Max & Min & Max & Min & Max & Inflex $^{+}$ \\
& $1)^{*}$ & $(2)$ & $(3)$ & 44 & 1 & $(2)$ & $(3)$ & 4 \\
\hline Temporal [ms] & 14 & 10 & 8 & 8 & 11 & 19 & 8 & 15 \\
Spatial [] & 1 & 3 & 1 & 2 & 2 & 3 & 2 & 2 \\
\hline
\end{tabular}

Notes: * numbers (1) to (4) correspond to the same points in Figure 2 ${ }^{+}$the last turning point for the shoulder was an inflexion 

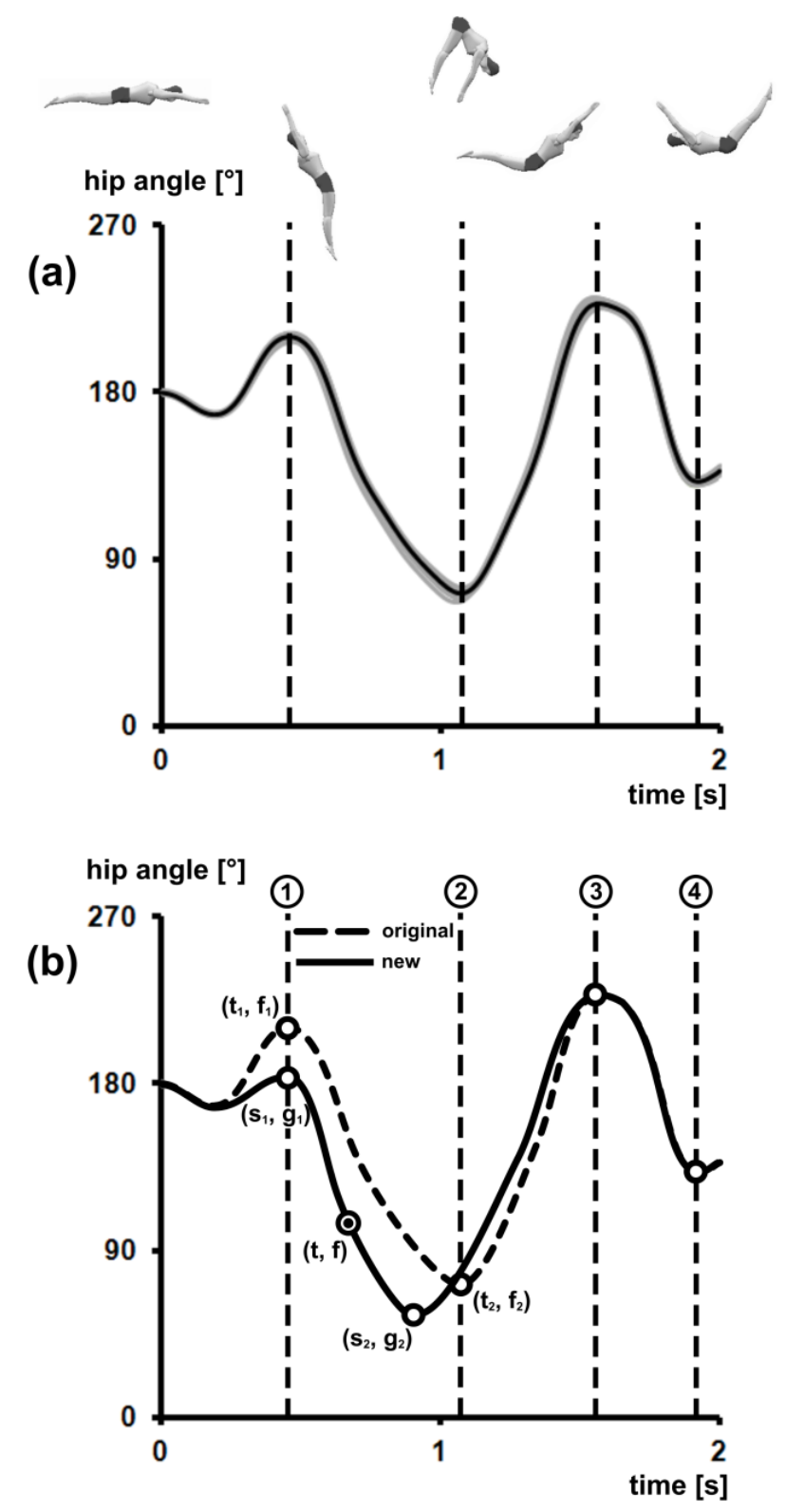

Figure 2. The joint angle history at the hip during the giant circles together with (a) the four extrema identified and (b) the method of transforming the splined data between consecutive extrema (black circle with white centres). The envelope generated from 20 perturbed joint angle histories is shown in grey in (a).

\section{Simulation model}

In order to determine release window for each trial and optimise the gymnast's technique a planar four-segment angle-driven model of a gymnast (comprising arm, torso, thigh and lower leg segments) and bar was used (Hiley \& Yeadon, 2003a). The bar and the gymnast's shoulder structure were modelled as damped linear springs (Figure 3). The spring at the shoulder represented the increase in length of the gymnast between the wrist and the hip. In addition to the shoulder spring, the torso segment was allowed to lengthen as the shoulder elevation angle increased (Begon, Wieber \& Yeadon, 2008). 


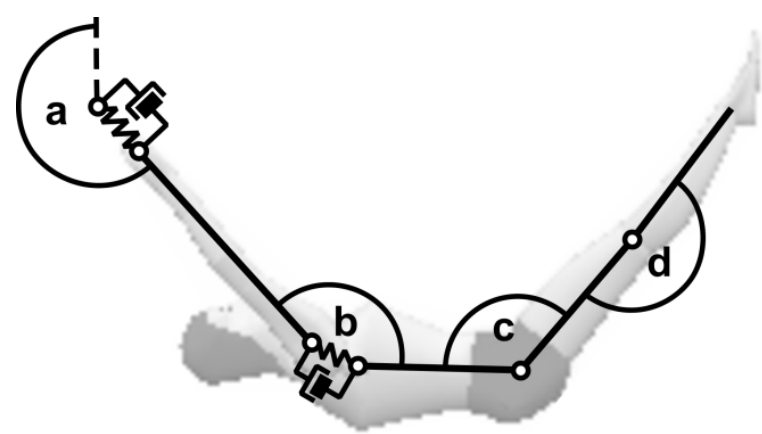

Figure 3. The four-segment gymnast - bar simulation model with damped springs representing bar and shoulder elasticity. Definitions of the (a) rotation, (b) shoulder, (c) hip and (d) knee angles.

Model parameters comprised segmental inertia data calculated from anthropometric measurements and Yeadon's (1990a) geometric inertia model (Table 2 ), stiffness and damping coefficients of the bar and shoulder springs, and the torso lengthening parameter were calculated using matching simulations. The initial conditions comprised the initial displacement and velocity of the bar, initial angular velocity of the arm, and initial orientation of the arm. Input to the model comprised the joint angle time histories of the shoulder, hip and knee in the form of quintic splines (Wood \& Jennings, 1979). Output from the model included the time histories of the horizontal and vertical bar displacements, the rotation angle (the angle between the upward vertical and the line from the neutral bar position to the model mass centre), the linear and angular momenta of the model about its mass centre, and the joint torque time histories. The equations of motion were derived using Newton's Second Law and by taking moments about the neutral (unloaded) bar position and the segment mass centres.

Table 2. Segmental inertia parameters and $T_{0}$ values used in the simulation model

\begin{tabular}{ccccccc}
\hline Segment & $\begin{array}{c}\text { Mass } \\
{[\mathrm{kg}]}\end{array}$ & $\begin{array}{c}\text { Length } \\
{[\mathrm{m}]}\end{array}$ & $\begin{array}{c}\text { MCD } \\
{[\mathrm{m}]}\end{array}$ & $\begin{array}{c}\text { MOI } \\
{\left[\mathrm{kg} \cdot \mathrm{m}^{2}\right]}\end{array}$ & $\begin{array}{c}\text { Joint } \\
\text { action }\end{array}$ & $\begin{array}{c}\mathrm{T}_{0} \\
{[\mathrm{Nm}]}\end{array}$ \\
\hline Arm $^{+}$ & 8.42 & 0.57 & 0.32 & 0.28 & Shoulder flexion $^{\dagger}$ & 280 \\
Torso & 36.42 & 0.52 & 0.17 & 1.77 & Shoulder extension $^{\dagger}$ & 430 \\
Thigh $^{+}$ & 16.68 & 0.40 & 0.17 & 0.24 & Hip flexion $^{\dagger}$ & 370 \\
Leg $^{+*}$ & 8.38 & 0.40 & 0.23 & 0.21 & Hip extension $^{\dagger}$ & 496 \\
& & & & & Knee extension $^{\dagger}$ & 602 \\
& & & & & Knee flexion $^{\dagger}$ & 210 \\
\hline
\end{tabular}

Notes: $M C D$ is the distance of the segment mass centre from the joint centre nearer the bar, $\mathrm{MOI}$ is the moment of inertia about the segment mass centre and $T_{0}$ is the maximum isometric torque

+ data for both limbs

* Leg refers to the shank and foot

${ }^{\dagger}$ data for both joints 


\section{Matching Simulations and Release Window}

For each of the 10 dismounts the release window was determined from a matching simulation using a previously described method (Hiley \& Yeadon, 2003b). The matching procedure was used to produce a common set of model parameters for each trial (i.e. bar and gymnast spring stiffness and damping coefficients, torso lengthening parameter and adjustments to the segmental inertia parameters). All matching simulations used the same set of model parameter values determined from concurrent matching optimisations, but with the initial conditions and joint angle time histories from the individual recorded performances. The start and end of the release window was defined such that the normalised angular momentum, horizontal velocity of the mass centre and the subsequent time of flight lay within three standard deviations of the mean values obtained from the 10 trials. The method of Yeadon (1990b) was used to determine the normalised angular momentum (straight somersaults per flight time) at the instant of release for each trial.

The matching simulations were on average able to match the rotation angle to $2^{\circ}$, the bar displacements to $0.01 \mathrm{~m}$ (root mean squared differences) and the linear and angular momenta at release to within $1 \%$ of the recorded trials (Figure 4). The stiffness and damping coefficients of the bar (horizontal and vertical) and shoulder springs were 18222, 25279, and $28347 \mathrm{~N} / \mathrm{m}$ and 28, 27, and $5467 \mathrm{Ns} / \mathrm{m}$, respectively. The range of release windows obtained from the matching simulations was $35-71 \mathrm{~ms}$ and the average combined joint torque at shoulder, hip and knee using equation 3 was $240 \mathrm{Nm}$ (Table 3). The shoulder hip and knee contributions to the average joint torque values were $63 \%, 28 \%$ and $9 \%$ respectively.

Table 3. The flight characteristics and release windows determined from the 10 double layout performances

\begin{tabular}{cccccc}
\hline Trial & $\begin{array}{c}\text { Flight } \\
\text { time } \\
{[\mathrm{s}]}\end{array}$ & $\begin{array}{c}\text { Angular } \\
\text { momentum* } \\
{[\mathrm{ss}]}\end{array}$ & $\begin{array}{c}\text { Travel }^{+} \\
{[\mathrm{m}]}\end{array}$ & $\begin{array}{c}\text { Release } \\
\text { window } \\
{[\mathrm{ms}]}\end{array}$ & $\begin{array}{c}\text { Average } \\
\text { torque }^{\dagger} \\
{[\mathrm{Nm}]}\end{array}$ \\
\hline 1 & 1.24 & 1.67 & 2.82 & 64 & 243 \\
2 & 1.25 & 1.65 & 2.84 & 64 & 232 \\
3 & 1.24 & 1.61 & 2.56 & 65 & 243 \\
4 & 1.24 & 1.63 & 2.71 & 45 & 236 \\
5 & 1.24 & 1.61 & 2.66 & 71 & 238 \\
6 & 1.24 & 1.62 & 2.84 & 57 & 246 \\
7 & 1.24 & 1.67 & 2.69 & 69 & 258 \\
8 & 1.22 & 1.59 & 2.55 & 58 & 236 \\
9 & 1.25 & 1.61 & 2.48 & 42 & 242 \\
10 & 1.24 & 1.61 & 2.57 & 35 & 226 \\
Mean & $\mathbf{1 . 2 4}$ & $\mathbf{1 . 6 3}$ & $\mathbf{2 . 6 7}$ & $\mathbf{5 7}$ & $\mathbf{2 4 0}$ \\
Stdev & $\mathbf{0 . 0 1}$ & $\mathbf{0 . 0 3}$ & $\mathbf{0 . 1 3}$ & $\mathbf{1 2}$ & $\mathbf{9}$ \\
\hline
\end{tabular}

* normalised to straight somersaults per flight time

+ horizontal distance of the mass centre from the bar on landing

$\dagger$ average total joint torque at the shoulder, hip and knee for matching simulations based using equation 3 

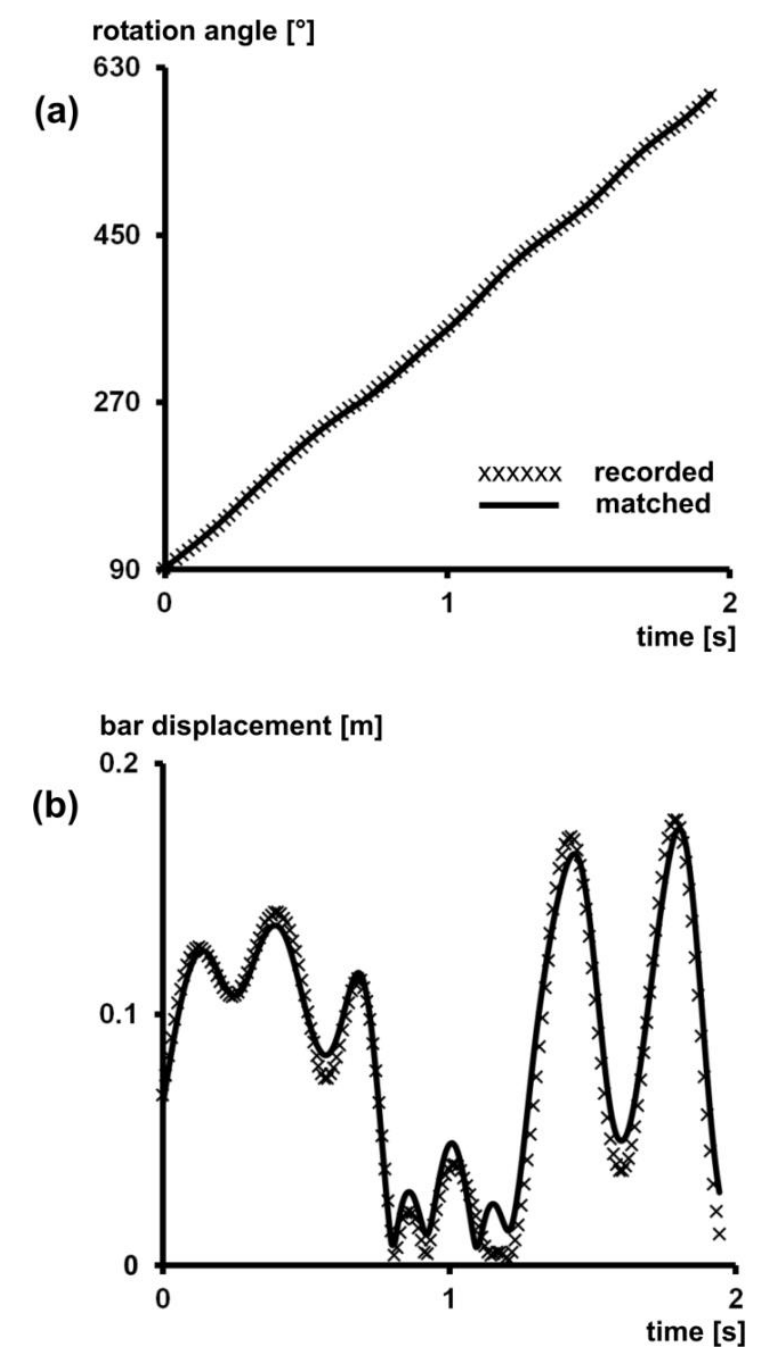

Figure 4. An example of a typical matching simulation (solid lines) compared with recorded performances (crosses) for: (a) rotation angle and (b) bar displacement.

\section{Optimisation}

Six optimisations were performed (three local and three global) to determine which criterion best characterised the technique used in the giant circles prior to release (i.e. minimising joint torque, maximising release window or maximising success in the presence of motor system noise). All simulations started from a rotation angle of $90^{\circ}$ (body horizontal), with initial angular velocity from the average trial, and ended once the rotation angle passed $630^{\circ}$. The procedure used a parallelised genetic algorithm that was run on a high performance computer with 60 processors (Carroll, 2001; van Soest \& Casius, 2003). In all optimisations the recommended optimisation algorithm tuning was used except for the selection of single point crossovers (Carroll, 2001). Single point crossovers were chosen as this was deemed appropriate for the application, since sections of joint angle time histories could then be passed on to the offspring rather than just single parameter values. The optimisation algorithm manipulated the parameters defining the joint angle time histories at the shoulder, hip and knee. The extremum (maximum and minimum) points in the mean joint angle time histories from the 10 recorded performances were used in the manipulation of these joint angle time histories (Figure 2b). The average data set of each time history was transformed between consecutive extrema in both the time and angle dimensions (Figure 2b). Any point (t, f) in the new data set (Figure 2b) could be calculated using transformations 1 and 2 . 


$$
\begin{aligned}
& t \rightarrow s_{1}+\left(\frac{t-t_{1}}{t_{2}-t_{1}}\right) \cdot\left(s_{2}-s_{1}\right) \\
& f \rightarrow g_{1}+\left(\frac{f-f_{1}}{f_{2}-f_{1}}\right) \cdot\left(g_{2}-g_{1}\right)
\end{aligned}
$$

where $t_{i}$ and $s_{i}$ are time parameters and $f_{i}$ and $g_{i}$ are angle parameters. In each joint angle time history (shoulder, hip and knee) parameters $s_{i}$ and $g_{i}$ for four extrema were varied by the optimisation algorithm (i.e. a total of 24 parameters for the three joints). An interpolating quintic spline was then fitted to the transformed data set to allow the calculation of the first and second derivative data required to drive the simulation model. The described method was used since it allowed more rapid changes between flexion and extension at a joint compared to the piecewise quintic function method used previously by the authors (Hiley \& Yeadon, 2003a). All joint angle histories were constrained using joint torque limits determined from isovelocity dynamometer measurements on the gymnast (Table 2) by fitting a function which expressed maximum voluntary torque in terms of joint angle and angular velocity (Forrester, Yeadon, King \& Pain, 2011; King, Kong \& Yeadon, 2009).

The optimisations were performed in two sets: one where the bounds placed on the joint angle time history parameters were set close to the mean values to determine whether the gymnast's technique occupied a local optimum and one where the bounds were set wide in order to find a global optimum. In the first set of optimisations the bounds on the mean joint angle time history parameters were set to \pm three standard deviations (SD) of the kinematic variability found at each turning point in the recorded trials (Table 1). In the second set of optimisations the bounds were set to $\pm 30^{\circ}$ or $\pm 0.10 \mathrm{~s}$ from the mean angle and time parameters respectively. If an optimal solution in the second set reached a bound, the optimisation was repeated using the current solution as a starting point and a new set of bounds set at $\pm 30^{\circ}$ or $\pm 0.10 \mathrm{~s}$.

Within each of the two sets of optimisations three optimisation criteria were used. The first criterion was based on minimising the mean total joint torque $F$ at the shoulder, hip and knee throughout the simulation (equation 3).

$$
F=\frac{1}{n} \sum_{i=0}^{n}\left(\left|T_{1}\right|+\left|T_{2}\right|+\left|T_{3}\right|\right)
$$

where $T_{1}, T_{2}$ and $T_{3}$ are the joint torques at the shoulder, hip and knee respectively and $i$ is the simulation integration step counter. The simulation was also required to produce a release window within the mean of the 10 trials $\pm 3 S D$ to ensure that a double layout somersault was possible (Table 3). The second criterion was based on maximising the size of the release window. Simulations exceeding the joint torque limits were given a large penalty. The third criterion was based on maximising the proportion of successful simulations despite operating in the presence of motor system noise. A successful simulation was one which produced a release window within the mean of the 10 trials \pm 3 SD and did not exceed the joint torque limits. During the third optimisation, the parameters defining the joint angle time histories of the shoulder, hip and knee were randomly perturbed, in both the time and angle dimension, to the level of the lowest kinematic variability measured in the gymnast performances (i.e. standard deviations of $8 \mathrm{~ms}$ and $1^{\circ}$ ). Perturbations were added to the joint angle time history parameters using a random number generator with a normal distribution (Figure 2a), since the recorded deviations were normally 
distributed. For each set of joint angle parameters produced by the optimisation algorithm, 500 randomly perturbed simulations were performed. Simulations were given a score of 1 for a successful simulation and 0 for an unsuccessful simulation, with the percentage of successful simulations out of 500 returned as the score for that set of parameters.

The root mean squared difference between the mean recorded and optimal joint angle time histories was calculated for the shoulder, hip and knee angles for each of the six solutions. In addition percentage success was assessed for each of the six optimal solutions using 500 randomly perturbed simulations, where success was defined as in the third optimisation criterion.

\section{RESULTS}

In the first set of local optimisations used to determine which criterion best characterised the gymnast's technique, the first optimisation (minimise joint torque criterion) was able to reduce the joint torques by $25 \%$ at the shoulder, hip and knee from a combined average of $240 \mathrm{Nm}$ in the average recorded trial (Table 3) to 180 $\mathrm{Nm}$ while still producing an adequate release window. The major contribution to reducing the joint torque came from the shoulder, accounting for $82 \%$ of the reduction ( $14 \%$ hip and $4 \%$ knee). The second optimisation was able to increase the size of the release window from a maximum of $71 \mathrm{~ms}$ in the gymnast trials (Table 3 ) to $129 \mathrm{~ms}$, an increase of $82 \%$. Over the 500 simulations used in each step of the third optimisation the solution produced $98 \%$ success despite the presence of noise in the joint angle time histories.

In the second set of optimisations used to find global solutions: the joint torque criterion was able to reduce the combined average torques at the shoulder, hip and knee to $134 \mathrm{Nm}$, again the major contribution to reducing the joint torque came from the shoulder, accounting for $92 \%$ of the reduction ( $7 \%$ hip and $1 \%$ knee). The release window criterion increased the window to $148 \mathrm{~ms}$ and the success criterion produced $100 \%$ success. Joint angle time histories for the global solutions deviated from the average recorded performance (Figure 5).

Table 4. Root mean squared differences in the joint angle time histories, bar displacements and rotation angle between the optimal solutions and the histories from the average recorded trial

\begin{tabular}{lcccccc}
\hline $\begin{array}{l}\text { Optimisation/ } \\
\text { Criterion }\end{array}$ & $\begin{array}{c}\text { Shoulder } \\
{\left[{ }^{\circ}\right]}\end{array}$ & $\begin{array}{c}\text { Hip } \\
{\left[{ }^{\circ}\right]}\end{array}$ & $\begin{array}{c}\text { Knee } \\
{\left[{ }^{\circ}\right]}\end{array}$ & $\begin{array}{c}\text { Bar } \\
{[\mathrm{m}]}\end{array}$ & $\begin{array}{c}\text { Rot angle } \\
{\left[{ }^{\circ}\right]}\end{array}$ & $\begin{array}{c}\text { Success } \\
{[\%]}\end{array}$ \\
\hline $\begin{array}{l}\text { Local } \\
\text { Min torque }\end{array}$ & 7 & 6 & 5 & 0.01 & 1 & 21 \\
Max window & 4 & 6 & 4 & 0.02 & 2 & 74 \\
Max success & 4 & 4 & 3 & 0.01 & 2 & 98 \\
Global & & & & & & \\
Min torque & 19 & 20 & 8 & 0.02 & 4 & 12 \\
Max window & 8 & 21 & 7 & 0.04 & 12 & 56 \\
Max success & 8 & 11 & 5 & 0.02 & 3 & 100 \\
\hline
\end{tabular}

The root mean squared (RMS) differences of the joint angle histories, bar displacements and rotation angle between the average recorded trial and the optimal solutions were all smaller for the local optimisations compared to the global optimisations (Table 4). The success of the six solutions ranged from $12 \%$ to $100 \%$ (Table 4). 
(a)

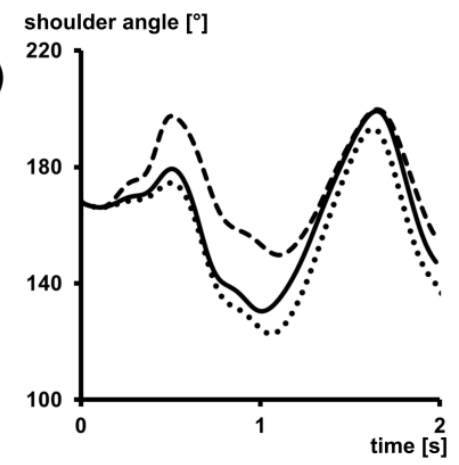

(b)

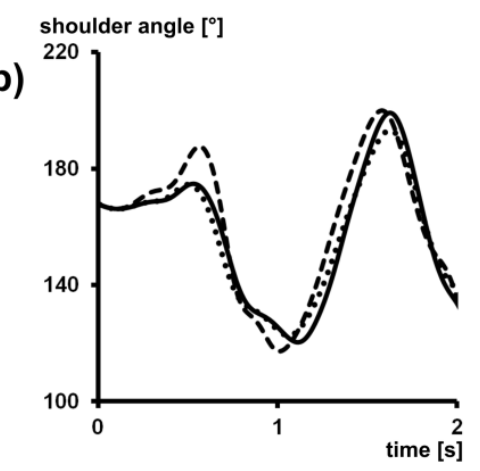

(c)

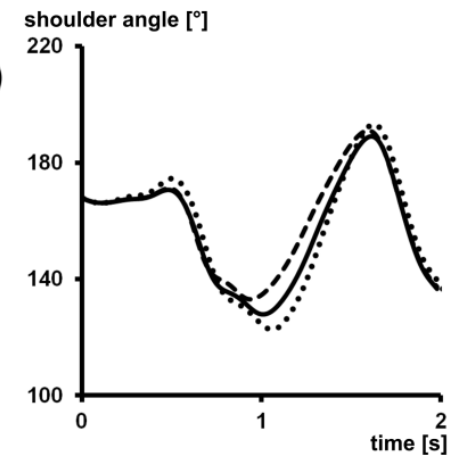

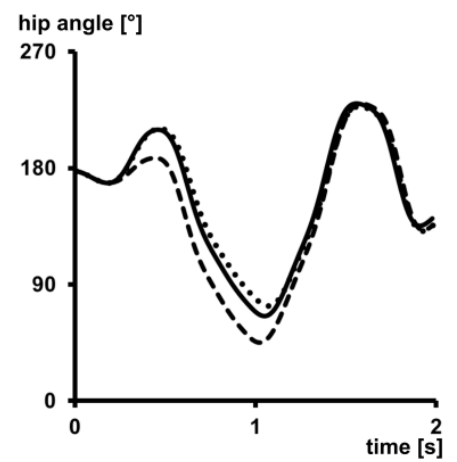
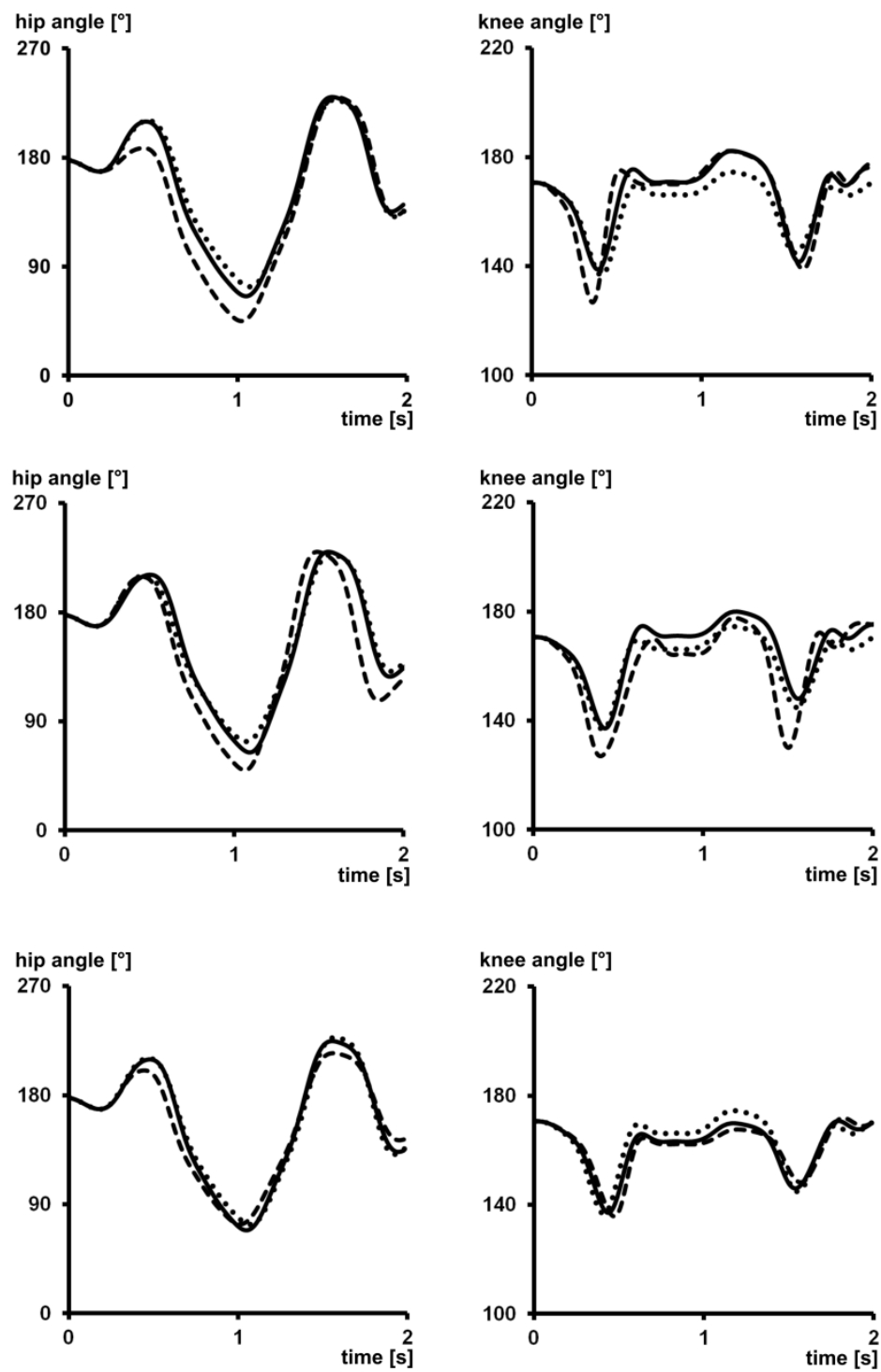

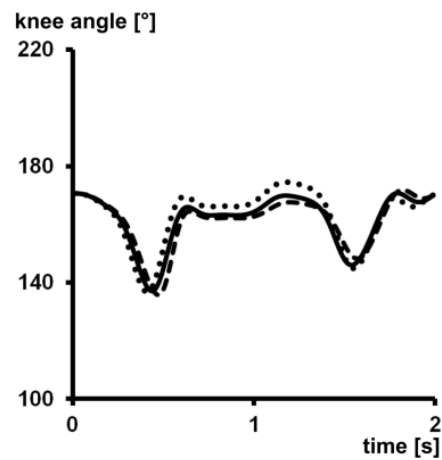

Figure 5. Shoulder, hip and knee joint angle time histories from the three local (black lines) and three global (dashed lines) optimal solutions for: (a) minimising joint torque, (b) maximising release window, and (c) maximising success in the presence of noise along with the average histories from the recorded performances (dotted lines).

\section{DISCUSSION}

The aim of the present study was to determine which optimisation criterion best characterised the technique adopted by an elite gymnast during giant circles prior to a dismount. The three optimisation criteria were allowed to find both local and global optima. In the case of minimising joint torque, the local optimisation reduced the average total joint torque by $25 \%$ and the global optimisation by $45 \%$. Although remaining within joint torque limits is an important constraint, it appears that the gymnast's technique cannot be characterised by this criterion, since both the local and global optimisations were able to reduce the joint torques substantially. When maximising the release window, the local optimisation was able to increase the window by $82 \%$ and the global optimisation by nearly $108 \%$ compared to the gymnast trials. Again it would appear that in the 10 recorded trials the gymnast was not attempting to maximise his release window, since both the local and global optimisations were able to increase the size of the release window substantially. However, when maximising success in the presence of noise the local optimisation 
was able to produce a $98 \%$ success rate, whilst the global optimisation produced a $100 \%$ success rate which was only a small improvement over the local optimum. It can therefore be concluded that the gymnast's technique has the characteristic of maximising success, since a local optimisation, close to the gymnast's technique, performed almost as well as a global optimisation. These success rates are consistent with the gymnast's performance of 10 successful dismounts from 10 attempts.

In the local optimum for maximising success, $2 \%$ of the dismounts were classified as unsuccessful due to an insufficiently large release window. Closer inspection of why these simulations failed showed that in half of them the normalised angular momentum fell just outside the bounds defining a successful window and in the other half the travel in flight dropped below the lower bound. Neither of these would have prevented a gymnast from completing a safe and successful dismount although the required changes in body shape may be penalised by judges in a competition.

In order to investigate the maximising success criterion it was necessary to determine the release window for the gymnast trials. The average release window ( $57 \pm 12 \mathrm{~ms}$ ) obtained from the repeated trials was smaller than the average from the 2000 Olympics high bar final (117 $\pm 26 \mathrm{~ms}$ from Hiley \& Yeadon, 2003b). The reason for this is that the ranges of release parameters defining the start and end of the release window for the individual gymnast in the present study were smaller than the ranges of the Olympic performances since these were determined from a group of eight gymnasts.

The aim of the study was not to find a composite criterion that accurately matched the joint angle time histories produced by the gymnast. If this had been the case an inverse optimal control approach could have been used to find the appropriate parameters and weightings which would produce a close match to the recorded performances (Mombaur, Truong \& Laumond, 2010). When such an approach is applied to recreating human movement patterns, components of the criterion are often related to producing smooth movements, which are a feature of human movement (Mombaur et al., 2010). Components within optimisation criteria which minimise joint torque, torque change, or jerk are often required to cope with inadequate or absent representations of human muscle and its innervation. In the present study the joint angle time histories were generated from human trajectories which were constrained by torque-angle-angular velocity profiles derived from measurements taken from the gymnast. In other words, by using the described methods, solutions will incorporate features associated with minimum torque change and jerk since they are the result of the mechanical constraints placed on the system by the properties of human muscle (Nakano, Imamizu, Osu, Uno, Gomi, Yoshioka \& Kawato, 1999). By modelling the structures responsible for the smooth nature of human movement the present study was able to investigate the overall, higher control strategy adopted by the gymnast.

The origin of the noise component of the kinematic variability is likely to be a result of noise within the neural system (Harris \& Wolpert, 1998), therefore it could be argued that a muscle-driven or torque-driven simulation model would have been more appropriate to address the current problem. However, by using an angle-driven model the noise added to the simulation was able to be based on measured values, both in magnitude and type of distribution, whereas determining the noise level and type of noise distribution in the many components of the neural system would be more problematic. Despite the model being termed "angle-driven", specifying the joint angle time histories specifies the joint angular accelerations. Since it is a 
mechanical system this also specifies the joint torques used by the model. Whatever the distribution of noise at the motor command level and within the motor system (which is unknown) the resulting noise at the kinematic level was found to be normally distributed (Hiley et al., 2013). Therefore, adding the noise at the kinematic level results in the torques required to have produced the perturbed movements.

The local optimisation performed in the presence of noise demonstrated that a solution space for maximising success lay close to the gymnast's technique. Nearly all of the 10 recorded trials fell within the envelope generated by adding three standard deviations of the kinematic variability determined from the recorded performances (Table 1) to each turning point of the local optimal solution (Figure 6). It may therefore be argued that the local optimal technique for maximising success and the gymnast's technique have a common solution space and that maximising success characterises the gymnast's technique. The fact that the gymnast's performances lie within a solution space defined by maximising success is consistent with Wolpert's (2007) hypothesis regarding the underlying strategy adopted for human movement.
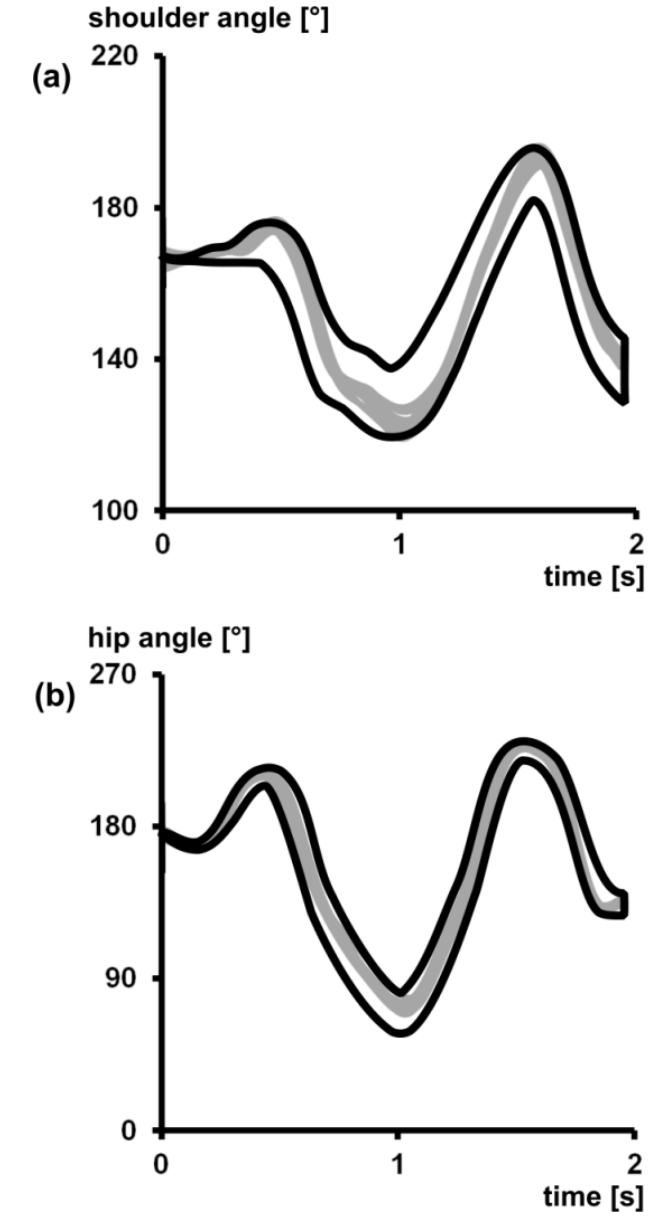

Figure 6. The (a) shoulder and (b) hip joint angle time histories from the 10 recorded performances (grey lines) along with the envelope generated by adding noise equivalent to three standard deviations of that reported kinematic movement variability in the actual performances (Table 1) at each turning point to the local optimal solution for maximising success in the presence of noise.

All six optimal solutions were tested to see how robust the techniques were (i.e. the success rate in 500 perturbed simulations was determined). Minimising torque or maximising release window in the absence of noise gave optimum solutions with poor success rates whereas optimising for success gave optimum solutions with excellent success rates (Table 4). Although it would be expected that the optimal 
solutions for maximising success would perform better on this aspect than the other two, the results demonstrate that, unless coping with the noise resulting from the motor system is accounted for within the optimisation procedure, solutions are not automatically robust to such noise (Table 4). Since human movement will always contain noise (Bartlett et al., 2007; Cohen \& Sternad, 2009) it is likely that coping with its effects are incorporated in the movement strategies adopted by humans (Harris \& Wolpert, 1998; Wolpert, 2007). Therefore, coping with noise should be included in optimisations if human-like solutions are to be found.

Ultimately, optimising for success determines the solution space defined by the constraints placed on the system. If the task is simple, that is, if it only has weak constraints, the solution space may be large (i.e. repeated success can be achieved with a number of techniques) and so the preferred technique might well be to adopt a minimal effort or energy strategy (e.g. walking). If the task is more complex, such as the double layout dismount (where the gymnast must remain within strength and anatomical limits, satisfy the release window requirements and have a technique that is insensitive to the noise resulting from the motor system) it might be that the solution space is so small that it effectively defines the gymnast's technique.

\section{REFERENCES}

Anderson, F.C., \& Pandy, M.G. (2001). Dynamic optimisation of human walking. ASME Journal of Biomechanical Engineering, 123, 381-390.

Bartlett, R., Wheat, J., \& Robins, M. (2007). Is movement variability important for sports biomechanists? Sports Biomechanics, 6, 224-243.

Begon, M., Wieber, P-B., Yeadon, M.R. (2008). Kinematics estimation of straddled movements on high bar from a limited number of skin markers using a chain model. Journal of Biomechanics, 41, 581-586.

Broderick, M.P., \& Newell, K.M. (1999). Coordination patterns in ball bouncing as a function of skill. Journal of Motor Behaviour, 31, 165-188.

Carroll, D.L. (2001). FORTRAN genetic algorithm driver. Downloaded from: http://cuaerospace.com/carroll.ga.html

Cohen, R.G., \& Sternad, D. (2009). Variability in motor learning: relocating, channeling and reducing noise. Experimental Brain Research, 193, 69-83.

Forrester, S.E., Yeadon, M.R., King, M.A., \& Pain, M.T.G. (2011). Comparing different approaches for determining joint torque parameters from isovelocity dynamometer measurements. Journal of Biomechanics, 44, 955-961.

Hamill, J., van Emmerik, R.E.A., Heiderscheit, B.C., \& Li, L. (1999). A dynamical systems approach to lower extremity running injuries. Clinical Biomechanics, 14, 297-308.

Harris, C.M., \& Wolpert, D.M. (1998). Signal-dependent noise determines motor planning. Nature, 394, 780-784.

Hiley, M.J., \& Yeadon, M.R. (2003a). Optimum technique for generating angular momentum in accelerated backward giant circles prior to a dismount. Journal of Applied Biomechanics, 19, 119-130.

Hiley, M.J., \& Yeadon, M.R. (2003b). The margin for error when releasing the high bar for dismounts. Journal of Biomechanics, 36, 313-319.

Hiley, M.J., \& Yeadon, M.R. (2005). Maximal dismounts from high bar. Journal of Biomechanics, 38, 2221-2227. 
Hiley, M.J. \& Yeadon, M.R. (2008). Optimisation of high bar circling technique for consistent performance of a triple piked somersault dismount. Journal of Biomechanics, 41, 1730-1735.

Hiley, M.J., \& Yeadon, M.R. (2012). The effect of cost function on optimum technique of the undersomersault on parallel bars. Journal of Applied Biomechanics, 28, 10-19.

Hiley, M.J., \& Yeadon, M.R. (2013). Investigating optimal technique in a noisy environment: application to the upstart on uneven bars. Human Movement Science, 32, 181-191

Hiley, M.J., Zuevsky, V.V., \& Yeadon, M.R. (2013). Is skilled technique characterized by high or low variability? An analysis of high bar giant circles. Human Movement Science, 31, 171-180.

James, C.R. (2004). Considerations of movement variability in biomechanics research. In Stergiou, N. (Ed.) Innovative analyses of human movement. Human Kinetics. Champaign, IL.

King, M.A., Kong, P.W., \& Yeadon, M.R. (2009). Determining effective subjectspecific strength levels for forward dives using computer simulations of recorded performances. Journal of Biomechanics, 42, 2672-2677.

Koschorreck, J., \& Mombaur, K. (2012). Modeling and optimal control of human platform diving with somersaults and twists. Optimization and Engineering, 13, 29-56.

Mombaur K., Truong A., \& Laumond J.P. (2010). From Human to Humanoid Locomotion - An inverse optimal control approach. Autonomous Robots, 28, 369-383.

Nakano, E., Imamizu, H., Osu, R., Uno, Y., Gomi, H., Yoshioka, T., \& Kawato, M. (1999). Quantitative examinations of internal representations for arm trajectory planning: minimum commanded torque change model. Journal of Neurophysiology, 8, 2140-2155.

Newell, K.M., \& Corcos, D.M. (1993). Issues in variability and motor control. In Newell, K.M., Corcos, D.M. (Eds.) Variability and motor control. Human Kinetics. Champaign, IL.

Ren, L., Jones, R.K., \& Howard, D. (2007). Predictive modelling of human walking over a complete gait cycle. Journal of Biomechanics, 40, 1567-1574.

van Beers, R.J., Haggard, P., \& Wolpert, D.M. (2004). The role of execution noise in movement variability. Journal of Neurophysiology, 91, 1050-1063.

van Soest, A.J., \& Casius, R.L.J. (2003). The merits of a parallel genetic algorithm in solving hard optimiszation problems. Journal of Biomedical Engineering, 125, 141-146.

Wolpert, D.M. (2007). Probabilistic models in human sensorimotor control. Human Movement Science, 26, 511-524.

Wood, G.A., \& Jennings, L.S. (1979). On the use of spline functions for data smoothing. Journal of Biomechanics, 12, 477-479.

Yamasaki, T., Gotoh, K., \& Xin, X. (2010). Optimality of a kip performance on the high bar: An example of skilled goal-directed whole body movement. Human Movement Science, 29, 464-482.

Yeadon, M.R. (1990a). The simulation of aerial movement - II. A mathematical inertia model of the human body. Journal of Biomechanics, 23, 67-74.

Yeadon, M.R. (1990b). The simulation of aerial movement - III: The determination of the angular momentum of the human body. Journal of Biomechanics, 23, 75-83. 